\title{
Probability Based Virtual Machines Placement for Green Data Center
}

\author{
Ye Heng-zhou ${ }^{1,2}$ and Li Tao-shen ${ }^{3}$ \\ ${ }^{1}$ College of Electrical Engineering, Guangxi University, Nanning 530004, China \\ ${ }^{2}$ College Of Information Science and Engineering, Guilin University Of \\ Technology, Guilin 541000, China \\ ${ }^{3}$ School of Information and Engineering, Guangxi University, Nanning 530004 \\ 1,22002018@glut.edu.cn; ${ }^{3}$ tshli@gxu.edu.cn
}

\begin{abstract}
Virtual Machine Placement (VMP) is regarded as an important criterion to improve resource utilization and reduce energy consumption for cloud data centers. The existing $V M P$ schemes simply set the VM resource requirements fixed values and ignore their fluctuation characteristics. Assuming normal distribution resource requirements, we firstly present a model for data centers based on a more accurate energy consumption model for single machine. Then, an effective genetic algorithm is adopted to solve this model. In the algorithm, some important issues, such as the number of population, fitness function and calculating method of energy consumption are discussed. In the end, we validate our method by experiments.
\end{abstract}

Keywords: virtual machines placement; green data center; normal distribution; genetic algorithm

\section{Introduction}

As the core infrastructure of cloud computing, data center has attracted a great deal of attention from academia and industry. According to Amazon's CEMS project [1], energy related cost amounts to $42 \%$ of the total budget of Amazon's data center. It can not only reduce costs, but also can reduce greenhouse gas emissions by reducing the energy consumption of the data center. Therefore, governments have attached great importance to the problem of data center power consumption. For example, China's new Industrial Energy Efficiency Plan demands that the new data center has a power usage effectiveness (PUE) less than 1.5. It was found that energy consumed by servers accounts for $52 \%$ of the total consumption within a data center according to Emerson Network Power's analysis [2]. Thus, adopting effective Virtual Machines Placement (VMP) techniques to improve the utilization ratio of data center resources and reduce energy consumption of the servers is an important way to achieve server green data center.

Recently, there exist some works to explore VMP problem. For Internet applications, [3] presents a semi-online automatic scaling VMP algorithm which takes into account the needs to maximize user satisfaction, minimize VM migration frequency and minimize energy cost. In order to consider the backup data placement and tasks allocation problem simultaneously, [4] proposes a new multi-objective bi-level programming model based on Map-Reduce applications to improve energy efficiency of servers. Research carried out by Li MF et al. [5] finds that there always exists the resource consumption overhead caused by the waiting of virtual machines for server resource scheduling when the number of virtual CPUs is more than the number of physical CPUs. Accordingly, they put forward a solution to reduce server resource violation probability by reserving a certain CPU resources for each virtual machine. In [6], in light of resource constraints (CPU, MEM, 
network bandwidth, etc.), a VMP method is explored to minimize the weighted average cost of network resources and host resources. These studies assume that VM demand for resources is a constant value. However, especially for outburst Internet applications, VM demand for related resources fluctuates frequently. Simply taking the maximum or average value will adversely affect resource utilization or Service Level Agreements (SLAs). In contrast to the methods to predict the load of hosts or CPUs [7-9], this article assumes that the VM resource requirements accord with normal distribution. Similar to our work, [10] only takes into account the probability distribution characteristics of bandwidth requirements and does not affect the energy consumption model of servers. What's more, its main contribution is to offer an on-line packing algorithm by which the number of servers required is within $(1+\varepsilon)(\sqrt{2}+1)$ of the optimum for any $\varepsilon>0$.

Compared to the previous approaches, the contributions of this work are summarized as follows:

- We take into consideration the probabilistic characteristics of the VM resource requirements, and on this basis, we model the VM placement problem for green data center as a variant of Multiple-choice Multi-dimension Knapsack Problem (MMKP).

- We design a genetic algorithm with adaptive populations and new fitness function to solve our model. We also discuss the method to calculate energy consumption of PMs to decrease computation complexity.

The rest of the paper is organized as follows. Section II describes the energy consumption optimization model for data center as a variant of Multiple-choice Multidimension Knapsack Problem (MMKP). In order to solve this model, an effective genetic algorithm based on specific-design genetic operators, fitness calculation method and calculating method of energy consumption is presented in section III. Section IV uses numerical experiments to evaluate the proposed algorithms by comparing energy consumption cost and SLA default rate. Finally, section V concludes the paper.

\section{Energy Consumption Optimization Model for Data Center}

In this section, we choose an energy model of PM for data center to quantify the relationship between energy consumption and utility of CPU and memory, and then formally define the VMP problem as a variant of MMKP.

\subsection{Energy Model of PM}

Energy of a PM associates with the utilization of multi-kind hardware resource, such as CPU, memory, hard disk, network card and so on. Relative research [11] has shown that there exists a kind of approximate linear relationship between energy consumption of a PM and its CPU utilization. However, with the changes in computer architecture, the relationship between energy and resource utilizations may not be linear. A more accurate nonlinear model which is more suitable to apply in energy-aware VMP problem is proposed by [12]. Through exploring a variety of regression analysis methods and analyzing experimental results, this model assumes that energy consumption mainly depends on the utilizations of CPU and memory. We choose the latter to describe energy model as (1), where $u_{1}, u_{2}$ represent the utilizations of CPU and memory, respectively, and $a_{0}, a_{j i}$ are some constants which can be determined by experiments.

$e(U)=a_{0}+\sum_{j=1}^{2} \sum_{i=1}^{3} a_{j i} \cdot u_{j}{ }^{i}$

Usually, $u_{1}$ and $u_{2}$ vary with loads. Thus, during the time interval $\left[t_{1}, t_{2}\right]$, the energy of a PM can be expressed as (2).

$E=\int_{t_{1}}^{t_{2}} e(U(t)) d t$ 
Considering the distribution characteristics of $u_{1}$ and $u_{2}$, during a long time interval $\left[t_{1}\right.$, $\left.t_{2}\right]$, the numerical integral method[13] to solve the energy of a PM can be very timeconsuming. In this work, expectations are used to replace integral effects to reduce the time complexity. Providing $u_{j} \sim \mathrm{N}\left(\mu_{j}, \sigma_{j}^{2}\right)$, we can obtain the following expression.

$$
\begin{aligned}
E & \approx E\left(a_{0}+\sum_{j=1}^{2} \sum_{i=1}^{3} a_{j i} \cdot u_{j}^{i}\right)=a_{0}+\sum_{j=1}^{2} \sum_{i=1}^{3} a_{j i} \cdot E\left(u_{j}^{i}\right) \\
& =a_{0}+\sum_{j=1}^{2}\left[a_{j 1} \mu_{j}+a_{j 2}\left(\mu_{j}^{2}+\sigma_{j}^{2}\right)+a_{j 3}\left(\mu_{j}^{3}+3 \mu_{j} \sigma_{j}^{2}\right)\right]
\end{aligned}
$$

\subsection{Energy Optimization Model for Data Center}

Assume that there are $N$ PMs in a data center, denoted as $\left\{s_{1}, s_{2}, \ldots, s_{N}\right\}, K$ is the number of resource types for each PM, and $C_{n}^{K}$ represents the capacity of source $k$ on PM $s_{n}$. Suppose that there are $M \mathrm{VMs}$, denoted as $\left\{v_{1}, v_{2}, \ldots, v_{M}\right\}$, which need to be assigned to these $N$ PMs, and each VM can only be assigned to one PM. Let $r_{m}^{K}$ which obeys normal distribution $N\left(\mu_{m}^{K}, \sigma_{m}^{K^{2}}\right)$ denotes requirement for resource $k$ on VM m. Let $P$ is an $M * N$ matrix of binary variables that represents a VM placement; the element $p_{m n}$ is 1 if $v_{m}$ is placed on host $s_{n}, 0$ otherwise. Then, $R_{n}^{K}$, the total requirement for resource $k$ on PM $s_{n}$ follows normal distribution $N\left(\sum_{i=1}^{M} \mu_{i}^{K} p_{i n}, \sum_{i=1}^{M} \sigma_{i}^{K^{2}} p_{i n}\right)$, and its utilization can be expressed as $R_{n}^{K} / C_{n}^{K}$. If $p^{k}$ denotes the overflow probability allowed for resource $k$ by SLA on PMs and $\mathrm{P}(X)$ indicates the probability of event $X$, energy optimization model for data center can be described as follows.

Objective: $\min E=\sum_{i=1}^{n} E_{i}$

Constraints:

$$
\begin{aligned}
& P\left(R_{n}^{k} \geq C_{n}^{k}\right) \leq p^{k}, n=1,2, \ldots, N, k=1,2,3, \ldots, K \\
& \sum_{i=1}^{n} p_{i j}=1, j=1,2, \ldots, M \\
& p_{i j} \in\{0,1\}, i=1,2, \ldots, N, j=1,2, \ldots, M
\end{aligned}
$$

\section{Genetic-Based Algorithm}

Our model is actually a variation of MMKP, and involves nonlinear factors such as probability distribution and higher powers. For this kind of problem, BestFit (BF) and its variants often can get a good solution quickly. However, with the increase of the dimension and the number of constraints, its effect will face more challenges. Another way of solving this problem is evolutionary algorithm, which is robust, adaptable and can get a satisfactory solution in most situations. In this section, a genetic-based algorithm is elaborated for implementing this model.

\subsection{Genome Encoding}

When using Genetic algorithm (GA) for solving a problem, how to encode the problem with a suitable genome is one of the most important. In this work, the genome is denoted by a vector of $M$ integers where $M$ is the number of VMs. This vector is arranged as $\left\langle d_{1}\right.$, 
$d_{2}, \ldots, d_{M}>$, where $d_{i} \in[1, N]$ is the index of the PM that VM $i$ is assigned to, and $N$ is the number of PMs.

\subsection{Genetic Operators}

The initial population is randomly generated except the one generated by BF. The size of population is the important factor affecting the effects of GA. The genetic algorithm with varying population size (GAVaPS) [14] method is adopted to determine the populations of next generation adaptively. Thus, the size of population after one iteration is defined by (4), where $\operatorname{AuxSize}(t)$ and $\operatorname{DeadSize}(t)$ are the number of genomes new created and died off during generation $t$ respectively.

$\operatorname{PopSize}(t+1)=\operatorname{PopSize}(t)+\operatorname{AuxSize}(t)-\operatorname{DeadSize}(t)$

For any genome, its age is initialized to 0 and increased by 1 each generation. And its lifetime is assigned by (5) to show that how many generations the genome can stay alive, where MinFit, MaxFit and AvgFit are the minimum, maximum and average fitness of the population, Fitness $[\mathrm{g}]$ is the fitness of genome $\mathrm{g}$, MinLT and MaxLT denote the minimum and maximum of the population, and $\alpha$ can be set to (MaxLT-MinLT)/2. When the age exceeds its lifetime, the genome will die.

$$
L T(g)= \begin{cases}\text { MinLT }+\alpha \frac{\text { Fitness }(g)-\text { MinFit }}{A v g F i t-M i n F i t} & \text { AvgFit } \geq \text { Fitness }(g) \\ \frac{\text { MaxLT }+ \text { MinLT }}{2}+\alpha \frac{\text { Fitness }(g)-\text { AvgFit }}{\text { MaxFit }- \text { AvgFit }} & \text { AvgFit }<\text { Fitness }(g)\end{cases}
$$

When mutation and crossover ratio are assigned to larger values, this scheme may cause the population to increase continually. Therefore, a threshold value MaxPop is used to limit the number of population. That is, when PopSize $(t+1)>$ MaxPop, part of individuals with lower fitness will be eliminated.

Given a genome, the mutation operator randomly selects an index of that array and replaces the corresponding value with a randomly alternative valid one.

For the crossover operator, an index is selected randomly used to partition each individual of the chosen pair of genomes into two segments. Two new genomes will be produced by switching the front segments of the old ones.

\subsection{Fitness Function}

A gene g represents one kind of VMP plan. Its fitness is determined by the energy consumption cost and penalty cost for violating constraints, as is shown in (6).

$$
\text { Fitness }(g)= \begin{cases}- \text { Energy }(g) & \mathrm{g} \text { is feasible } \\ -(\text { Energy }(g)+\text { Penalty }(g)) & \mathrm{g} \text { is not feasible }\end{cases}
$$

Energy $(\mathrm{g})$ can be calculated according to the model formulated in the previous section, where $p_{m n}=1$ if $d_{m}=n$, otherwise, $p_{m n}=0$.

The following rules should be taken into account to calculate Penalty $(\mathrm{g})$. The more the number of violation of constraints, the bigger the value of Penalty $(\mathrm{g})$. The heavier the degree of violation of constraints, the bigger the value of Penalty(g). Penalty(g) should have a similar magnitude to Energy (g). Therefore, Penalty (g) is designed as (7), where $\mathrm{E}^{*}$ is the estimate of energy consumption for data center and can be assigned to the mean of energy of the initial population.

$\operatorname{Penalty}(g)=\frac{E^{*}}{N K} \sum_{k=1}^{K} \sum_{n=1}^{N} \frac{N N S\left(P\left(R_{n}^{k} \geq C_{n}^{k}\right), p^{k}\right)}{p^{k}}$ 
$N N S(x, y)= \begin{cases}x-y & \mathrm{x}>\mathrm{y} \\ 0 & \mathrm{x} \leq \mathrm{y}\end{cases}$

\section{Experiments and Analysis}

As far as we know, the existing works about VMP assume that the VM requirements for resources are fixed values. In order to verify the necessity of considering the normal distribution characteristic of resource requirement, we compare our case with the cases when the resource requirements are taking fixed values (mean and maximum) with the consideration of the minimum number of PMs required, energy and SLA default rate. On the other hand, we select BF and MBFD[15] to compare with our GA.

\subsection{Parameter Values and Experimental Environment}

Without loss of generality, only four resources (CPU, memory, hard disk and network bandwidth) with corresponding $k$ value of 0 to 3 respectively are considered. Set $p^{k}=0.01$ and other parameters about PM and VM list in table 1. We set parameters associated with genetic algorithm in this paper as follows: PopSize $=200$, MaxSize $=1000$, mutation rate $=0.3$, crossover rate $=0.7$, iterative times $=M * 5$, and $N=M / 4$. We chose java1.7 to implement the proposed genetic algorithm and all experiments were performed on a 2.4 $\mathrm{GHz}$ Intel Xeon dual-core processor system with 4 GB RAM running Win7. Statistical rule of SLA default rates is as follows. In one test, if there is some resource on some PM in excess of the corresponding overflow ratio, this test is considered in violation of the SLA. And in 1000 times tests, the number of defaults divided by 1000 is referred to as the default rate. We ran every test 20 times and took the average.

Table 1. Values of Corresponding Parameters

\begin{tabular}{|l|l|l|l|}
\hline parameters & values & parameters & values \\
\hline$C_{n}^{0}$ & $3720 \mathrm{MPIS}$ & $\mu_{m}^{0}$ & {$[500,800]$} \\
\hline$C_{n}^{1}$ & $4 \mathrm{~GB}$ & $\mu_{m}^{1}$ & {$[400,600]$} \\
\hline$C_{n}^{2}$ & $500 \mathrm{~GB}$ & $\mu_{m}^{2}$ & {$[20,40]$} \\
\hline$C_{n}^{3}$ & $1000 \mathrm{Mb}$ & $\mu_{m}^{3}$ & {$[100,140]$} \\
\hline$\sigma_{m}^{0}$ & {$[50,80]$} & $\sigma_{m}^{2}$ & {$[2,4]$} \\
\hline$\sigma_{m}^{1}$ & {$[40,60]$} & $\sigma_{m}^{3}$ & {$[10,14]$} \\
\hline
\end{tabular}

\subsection{Benefits from Probability Distribution}

Fig. 1(a) compares the SLA default rates when VM requirements for resources take random (normal distribution), mean and maximum (3- $\sigma$ principle) values respectively. As can be seen from the figure, in the first and third cases, default rates are close to zero, while for the second case, default rate is more than $40 \%$ and increases with the number of VMs. SLA default rate is usually regarded as a kind of hard target, thus the scheme to take mean values is not desirable. 


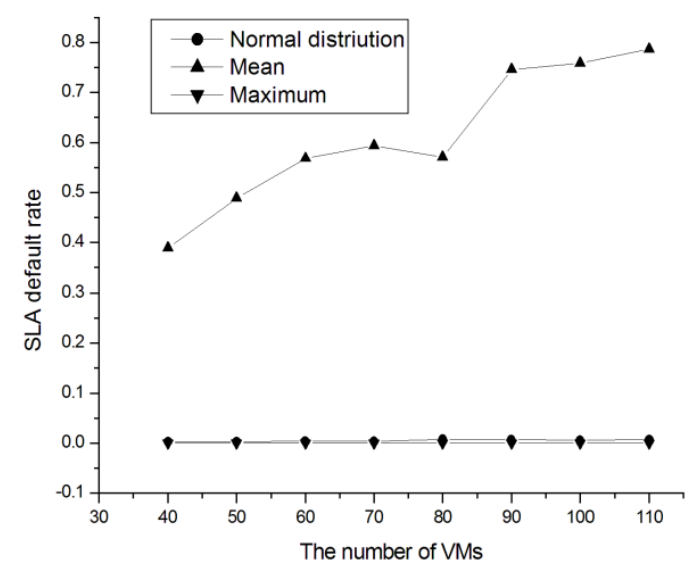

(a)SLA Default Rate

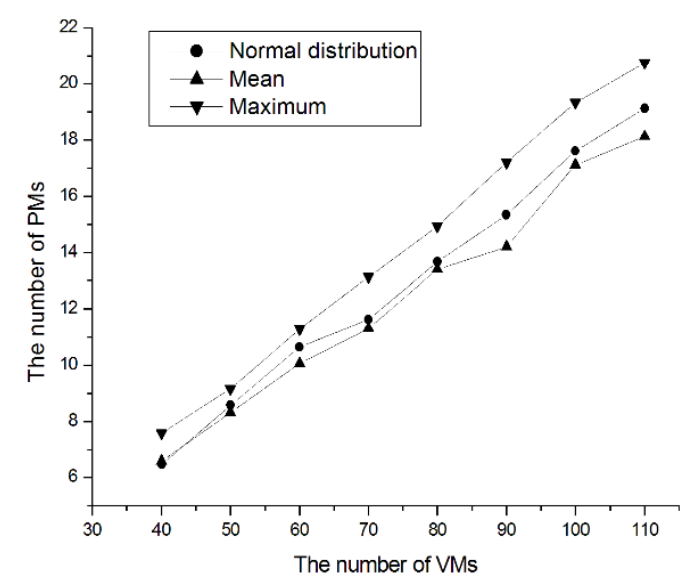

(b) Number of PMs

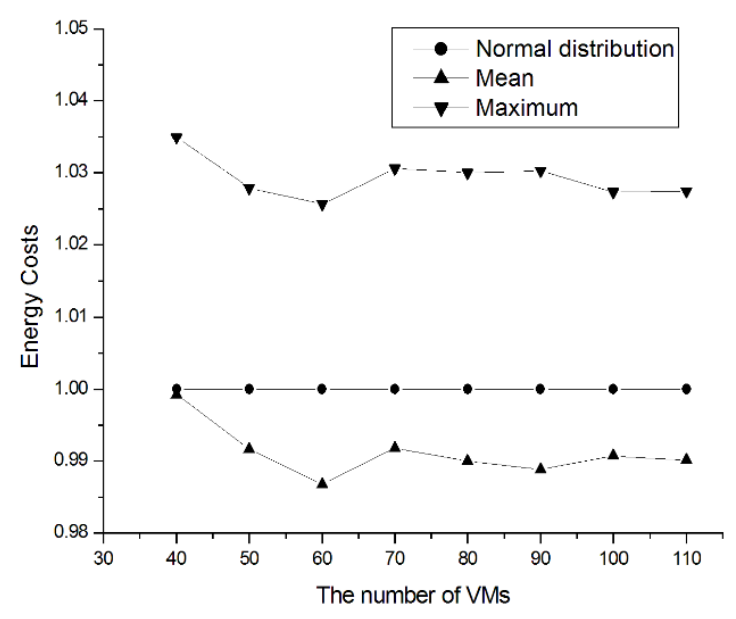

(c)Energy Costs

Figure 1. Comparison of SLA Default Rate, the Number of PMs and Energy

The comparison of number of PMs and energy is illustrated by Fig. 1(b) and (c) respectively in the same three circumstances. Compared to the maximum situation, the random one reduces the number of PMs by $10 \%$ and energy cost by $3 \%$. Hence, normal distribution model can obtain better VMP scheme. There are three reasons that optimizations about the number of PMs and energy are not very significant. (1) The optimal solution cannot always be obtained by genetic algorithm. (2) Due to the VM resource requirements with random values, resource utilization of PMs generally does not 
achieve the maximum even when the optimal solution is applied. (3) Our model considers idle overhead, i.e., when its utilization is zero, the PM still consumes energy. This is the reason why optimization effect of the number of PMs is more significant than energy. Considering the following case: all of VMs have the same resource utilization, and $r_{m}^{0} \sim N\left(532,68^{2}\right), r_{m}^{1} \sim N\left(400,80^{2}\right), r_{m}^{2} \sim N\left(50,10^{2}\right), r_{m}^{3} \sim N\left(100,20^{2}\right)$, the optimization effect of the number of PMs and energy will be shown as Fig. 2. The random scheme reduces the number of PMs by $40 \%$ and energy by $6 \%$ than the maximum one. If idle overhead is ignored, the expected optimization effect of energy will be remarkable.

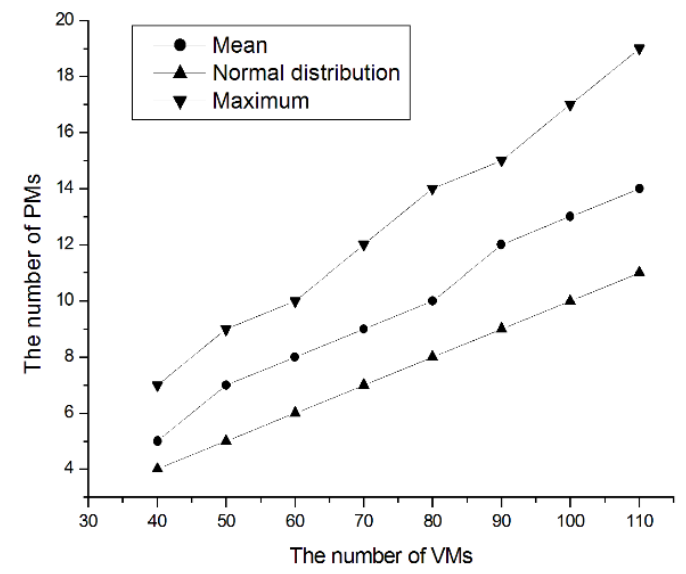

(a)Number of PMs

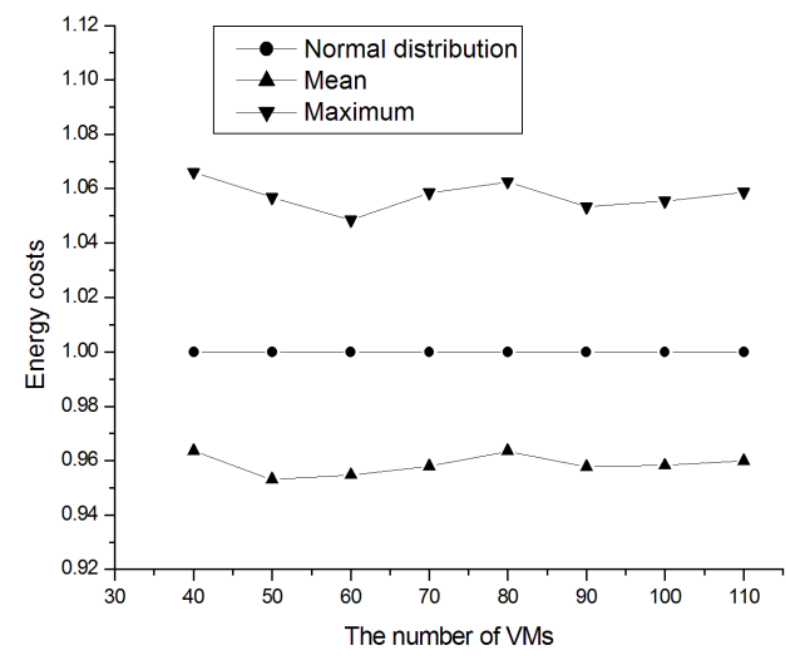

(b)Energy Costs

Figure 2. Comparison of the Number of PMs and Energy for a Special Case

\subsection{Effectiveness of GA}

Energy consumption and CPU utilization are important indicators of measuring the effectiveness of an algorithm. Fig. 3 and Fig. 4 show the energy and CPU utilization for various types of algorithms, which indicates that the effectiveness of GA is a little better than BF and MBFD. Although GA is more time-consuming, it is appropriate as an offline scheduling algorithm. 


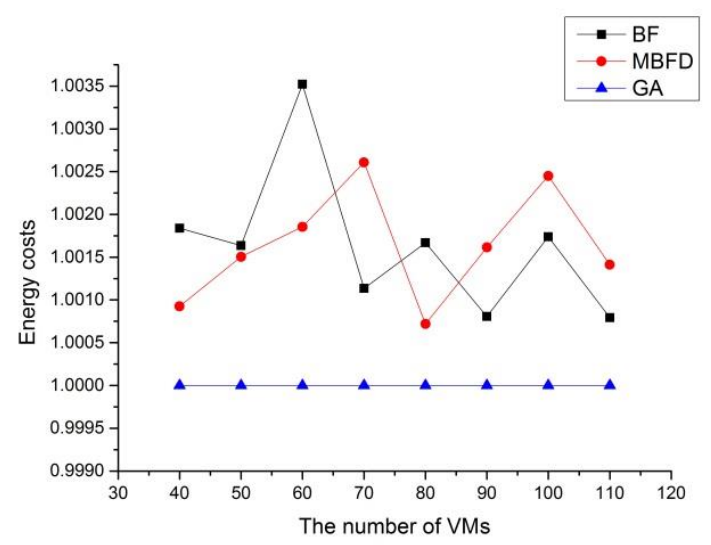

Figure 3. Energy for Various Types of Algorithms

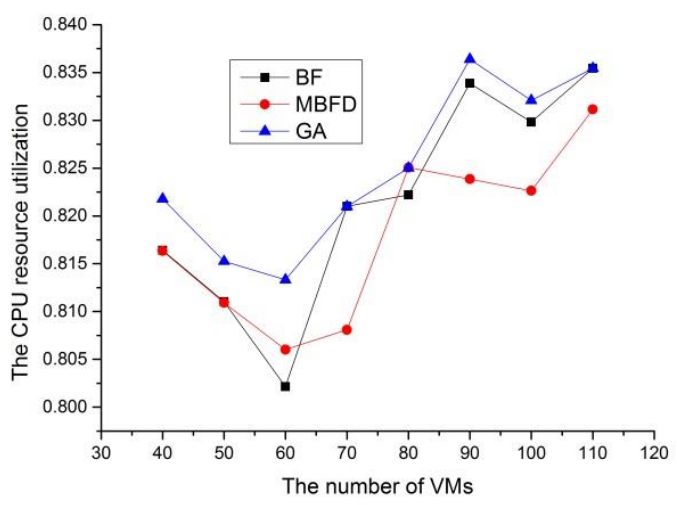

Figure 4. CPU Utilization for Various Types of Algorithms

\section{Conclusion}

The searching of optimal solution for VMP under SLA constraints to reduce energy of PMs is one of the effective ways to realize green data center. Our research shows that a more ideal solution can be expected if we consider the VMP from the angle of probability when the VM resource requirements are in accordance with normal distribution instead of a fixed value. The next move is to research the probability distribution of resource requirements of VMs and to explore VMP scheme when energy of both PMs and network devices are involved.

\section{Acknowledgment}

This work was supported by National Natural Science Foundation of China (No. 51365010) and National Natural Science Foundation of Guangxi (No. 2014GXNSFBA118269).

\section{References}

[1] J. Hamilton, "Cooperative expendable micro-slice servers (CEMS): low cost, low power servers for Internet-scale services", Proceedings of the 4th Biennial Conference on Innovative Data Systems Research (CIDR), Asilomar, California, (2009), pp.1-3.

[2] E. N. Power, "Energy logic: Reducing data center energy consumption by creating savings that cascade across systems", in A White Paper from the Experts in Business-Critical Continuity, (2008), pp.1-19. 
[3] Z. Xiao, Q. Chen and H. P. Luo, "Automatic scaling of Internet applications for cloud computing services", IEEE Transactions on computers, vol. 63, no. 5, (2014), pp.1111-1123.

[4] X. L. Wang, Y. P. Wang and Y. Cui, "A new multi-objective bi-level programming model for energy and locality aware multi-job scheduling in cloud computing", Future Generation Computer Systems, vol. 36, (2014), pp. 91-101.

[5] M. F. Li, J. P. Bi and Z. C. Li, "Resource-Scheduling-Waiting-Aware virtual machine consolidation", Journal of Software, vol. 25, (2014), pp. 1388-1402.

[6] J. K. Dong, X. Jin, H. B. Wang, Y. Y. Li, P. Zhang and S. D. Cheng, "Energy-Saving virtual machine placement in cloud data centers", Proceedings of the 13th IEEE/ACM International Symposium on Cluster, Cloud \& Grid Computing (CCGrid), Delft, (2013), pp. 618-624.

[7] Q. P. Yang, C. L. Peng, H. Zhao, Y. Yu, Y. Zhou, Z.Q. Wang and S. D. Du, "A new method based on PSR and EA-GMDH for host load prediction in cloud computing system", The Journal of Supercomputing, vol. 68, no. 3, (2014), pp.1402-1417.

[8] D. Y. Yang, J. Cao, C. Yu and J. Xiao, "A multi-step-ahead CPU load prediction approach in distributed system", The Second International Conference on Cloud and Green Computing (CGC), Xiangtan, China, (2012), pp. 206-213.

[9] S. Di, D. Kondo and W. Cirne, "Host load prediction in a Google compute cloud with a Bayesian model", The International Conference for High Performance Computing, Networking, Storage and Analysis(SC), Salt Lake City, UT, (2012).

[10] M. Wang, X. Q. Meng and L. Zhang, "Consolidating virtual machines with dynamic bandwidth demand in data centers", Proceedings of the IEEE INFOCOM, Shanghai, China, (2011), pp. 1-75.

[11] M. Armbrust, A. Fox, R. Griffith, A. D. Joseph, R. Katz and A. Konwinski, "A view of cloud computing", Communications of the ACM, vol. 53, no. 4, (2010), pp.50-58.

[12] L. Luo, W. J. Wu and F. Zhang, "Energy modeling based on cloud data center", Journal of Software, vol. 25, no. 7, (2014), pp. 1371-1387.

[13] Z. P. Liu, S. G. Wang, Q. B. Sun and F. C. Yang, "Energy-aware intelligent optimization algorithm for virtual machine replacement", Journal of Huazhong University of Science \& Technology (Nature Science), vol. 40, (2012), pp. 398-402.

[14] J. Arabas, Z. Michalewicz and J. Mulawka, "GAVaPS-a genetic algorithm with varying population size", Proceedings of the 1st IEEE Conference on Evolutionary Computation, Orlando, FL, (1994), pp. 73-78.

[15] A. Beloglazov, J. Abawajy and R. Buyya, "Energy-aware resource allocation heuristics for efficient management of data centers for cloud computing", Future Generation Computer Systems, vol. 28, no. 5, (2012), pp. 775-768.

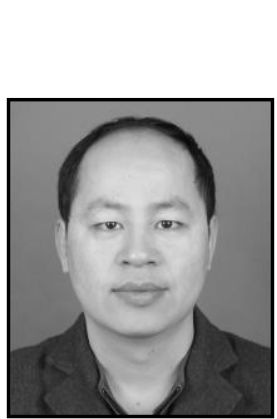

\section{Authors}

HengZhou Ye, He received the B.S. and M.S. degrees in Guilin Institute of Technology, P. R. China, in 2002 and 2007, respectively. Now, he is an associate professor at the College of Information Science and Engineering, Guilin University of Technology and is pursuing the PH.D. degree at Guangxi University. His current research interests include cloud computing and service composition.

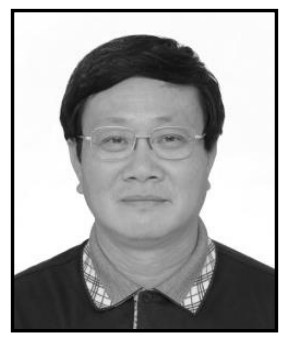

TaoShen Li, He received the B.S., M.S. and Ph.D. degrees in computer science and technology from the Central South University, P. R. China, in 1982, 1989 and 2008, respectively. He is currently a Professor of the School of Computer, Electronics and Information at Guangxi University in P. R. China. His research interests are in the area of cloud computing, wireless mesh networks, distributed database, network and information security. 
International Journal of Database Theory and Application Vol.9, No.12 (2016) 